\title{
Hyaluronic Acid Coated Liposomes Co-Delivery of Natural Cyclic Peptide RA-XII and Mitochondrial Targeted Photosensitizer for Highly Selective Precise Combined Treatment of Colon Cancer
}

\author{
Yanqing $\mathrm{Xu}\left(\mathbb{D}^{*}\right.$ \\ Yongrong Yao* \\ Linxiao Wang \\ Huachao Chen (ID \\ Ninghua Tan (D)
}

State Key Laboratory of Natural Medicines, School of Traditional Chinese Pharmacy, China Pharmaceutical University, Nanjing, 21 I 198, People's Republic of China

*These authors contributed equally to this work
Correspondence: Ninghua Tan; Huachao Chen

State Key Laboratory of Natura

Medicines, School of Traditional Chinese Pharmacy, China Pharmaceutical

University, Nanjing, 21 I 198, People's

Republic of China

Tel/Fax +862586/85772

Email nhtan@cpu.edu.cn; huachao.

chen@cpu.edu.cn
Background: Natural cyclopeptide RA-XII, isolated from Rubia yunnanensis, is a promising chemotherapeutic agent for colon cancer. The photosensitizer protoporphyrinIX attached with triphenylphosphonium (TPP) could possess mitochondria targeting capacity and exert photodynamic therapy (PDT) by inducing oxidizing damage to the mitochondria and cell apoptosis eventually. In this work, $\mathrm{pH}$-sensitive liposomes were constructed to simultaneously deliver RA-XII as a chemotherapeutic drug and modified porphyrin as a mitochondria-targeting photosensitizer to treat colon cancer, and verified its mechanism of action and antitumor therapeutic efficacy.

Methods: The colon cancer targeting liposome nanoparticle RA/TPPP-Lip was synthesized using thin film hydration. The therapeutic effect and targeting ability of RA/TPPP-Lip was investigated in vitro. And use HCT116 cell allogeneic subcutaneous transplantation tumor model to investigate the anti-tumor and targeting effects of RA/TPPP-Lip in vivo.

Results: RA/TPPP-Lip gained the targeting ability through surface-modified HA to increase the accumulation of RA-XII and TPPP in colon cancer cells. A series of in vitro experimental results showed that TPPP produced cytotoxic ROS under laser irradiation to directly damage cell mitochondria and played a combined role with RA-XII, making RA/TPPP-Lip the best colon cancer cell growth inhibitory effect. Furthermore, in vivo antitumor experiments showed that the RA/TPPP-Lip substantially accumulated at the tumor site and efficiently repressed tumor growth in nude mice.

Conclusion: We have successfully designed a new cancer-targeted nanomedicine platform (RA/TPPP-Lip) for the collaborative treatment of colon cancer, which can achieve the targeted continuous release of multiple therapeutic drugs. This work provides a new strategy for precise combination therapy, which may promote the further development of collaborative cancer treatment platforms.

Keywords: natural cyclic peptide, liposome, mitochondrial targeted, photosensitizer, combination therapy nanosystem, colon cancer

\section{Introduction}

Colon cancer is one of the common malignancy of digestive tract in the world and the second leading cause of cancer associated death in the West. ${ }^{1,2}$ Chemotherapy still plays a significant role in the management of colon cancer because colon cancer is an advanced cancer that is difficult to completely eradicate through surgery. ${ }^{3}$ However, traditional cancer chemotherapeutic drugs have showed poor antitumor activity on 
account of their major limitations, including low solubility and the lack of tumor specificity. ${ }^{4}$ In recent years, natural compounds, especially naturally derived compounds, have been recognized as effective anticancer drugs to improve efficacy and reduce the side effects of chemotherapy, at least $60 \%$ of the available anti-cancer agents currently on the market can be traced to natural products. ${ }^{5}$ Thereinto, increasing interest has been focused on cyclic peptides and related congeners due to their unique structures and antitumor activity. Natural cyclopeptide RA-XII, isolated from Rubia yunnanensis, demonstrates anti-carcinogenic and antiinflammatory properties. ${ }^{6,7}$ Our previous investigation has established that RA-XII suppressed protective autophagy for promoting apoptosis in liver cancer cells through AMPK/mTOR/P70S6K pathway, and had potent efficacy in the treatment of breast cancer via PI3K/AKT/NF- $\mathrm{KB}$ pathway. ${ }^{8-11}$ Moreover, our latest study demonstrated that RA-XII inhibited tumor growth and metastasis in colon cancer, indicating that it is a promising chemotherapeutic agent for colon cancer. ${ }^{12,13}$

Present chemotherapy based on single-drug regimens is far from perfect. Cancer monotherapy treatment has severe side effects at higher doses and leads to multi-drug resistance, at last resulting in chemotherapy failure. Owing to the complex components and unique properties of tumor microenvironment, a combination of therapeutic approaches with two or more different drugs or mechanisms offers great promise for effective cancer treatment, due to its superiority in reducing non-specific toxicities, overcoming multi-drug resistance, improving prognosis and achieving synergistic effects. ${ }^{14-17}$ It is argued that multidrug combination therapy has inherent pharmacological and pharmacokinetic advantages compared with single-agent therapy, because cancer development often involves many contributing factors that hitting a single target may not be enough to treat it efficiently. Photodynamic therapy (PDT) typically uses photosensitizers (PSs), such as porphyrins and phthalocyanines, absorbs light in a wavelength range of $600-800 \mathrm{~nm}$ and then forms cytotoxic reactive oxygen species (ROS), such as singlet oxygen and hydroxyl radicals, which may cause cancer cell death eventually. The use of light-induced enhancement of drug deposition is an emerging field of research: PDT has attracted widespread attention due to the noninvasive nature, sitespecific triggered drug release, controllability, and insignificant side effects. ${ }^{18-21}$ Based on the excellent antitumor effects of PDT, the combination of both chemotherapy and PDT into a single therapeutic modality may achieve great synergistic therapeutic effect in vivo and significantly improve antitumor efficacy. ${ }^{22,23}$ In addition, targeted phototherapy at the organelle level (nucleus, ${ }^{24,25}$ mitochondria, ${ }^{26}$ endoplasmic reticulum, ${ }^{27}$ and plasma membrane $)^{28,29}$ can address many important issues in the field of cancer therapy and improve the efficiency of PDT. Mitochondria, one of the most important energy-generating organelles, is crucial to maintain the cell homeostasis, so the direct damage of them could can lead to cell apoptosis rapidly. More importantly, mitochondria-targeting photosensitizers can overcome hypoxia factor and mitochondria provides excellent locations for organ-specific phototherapy because of its sensibility to ROS and heat. ${ }^{30-32}$ So far, many methods to achieve mitochondrial targeting have been reported. Triphenylphosphonium (TPP), a cation rich in delocalized positive charge, has sufficient lipophilicity. ${ }^{26}$

We have successfully developed a mitochondrial-targeted photosensitizer: Protoporphyrin-IX conjugated with TPP ligand (TPPP), which could easily permeate lipid bilayers and quickly accumulate into mitochondria (Scheme 1). Under the excitation of Near-infrared light at $660 \mathrm{~nm}$ wavelength, the TPPP in mitochondria kill colon cancer cells through ROS generation in situ. To avoid multiple administrations of different therapeutic agents and accumulation of RA-XII and TPPP in healthy cells, we developed a multistage responsive smart nanomedicine platform that possesses both chemotherapy and PDT therapeutic functions. In our design, RA-XII and TPPP were either embedded into the $\mathrm{pH}$-sensitive liposomes shell to prolong the systematic circulation of antitumor agents. To improve colon tumor targetability, hyaluronic acid (HA) has been coated on the liposome surface, since HA can bind specifically to CD44, which is overexpressed in colon cancer cells and increase cellular uptake through CD44-mediated endocytosis. ${ }^{33-36}$ The obtained RA/TPPP-Lip nanoparticles could be degraded by the acidic microenvironment and release of RA-XII and TPPP. After laser irradiation, TPPP could lead to oxidative damage of mitochondrial through the generation of cytotoxic ROS and then kill the colon cancer cells in coordination with RA-XII. We have demonstrated that this system exhibited promising antitumor effect with reduced systemic adverse effect in both in vitro and in vivo assays.

\section{Materials and Methods}

\section{Materials}

Lipoid S100 (Soya Phosphatidvl Chdiae) was purchased from Lipoid (Ludwigshafen, Germany). Cholesterol 


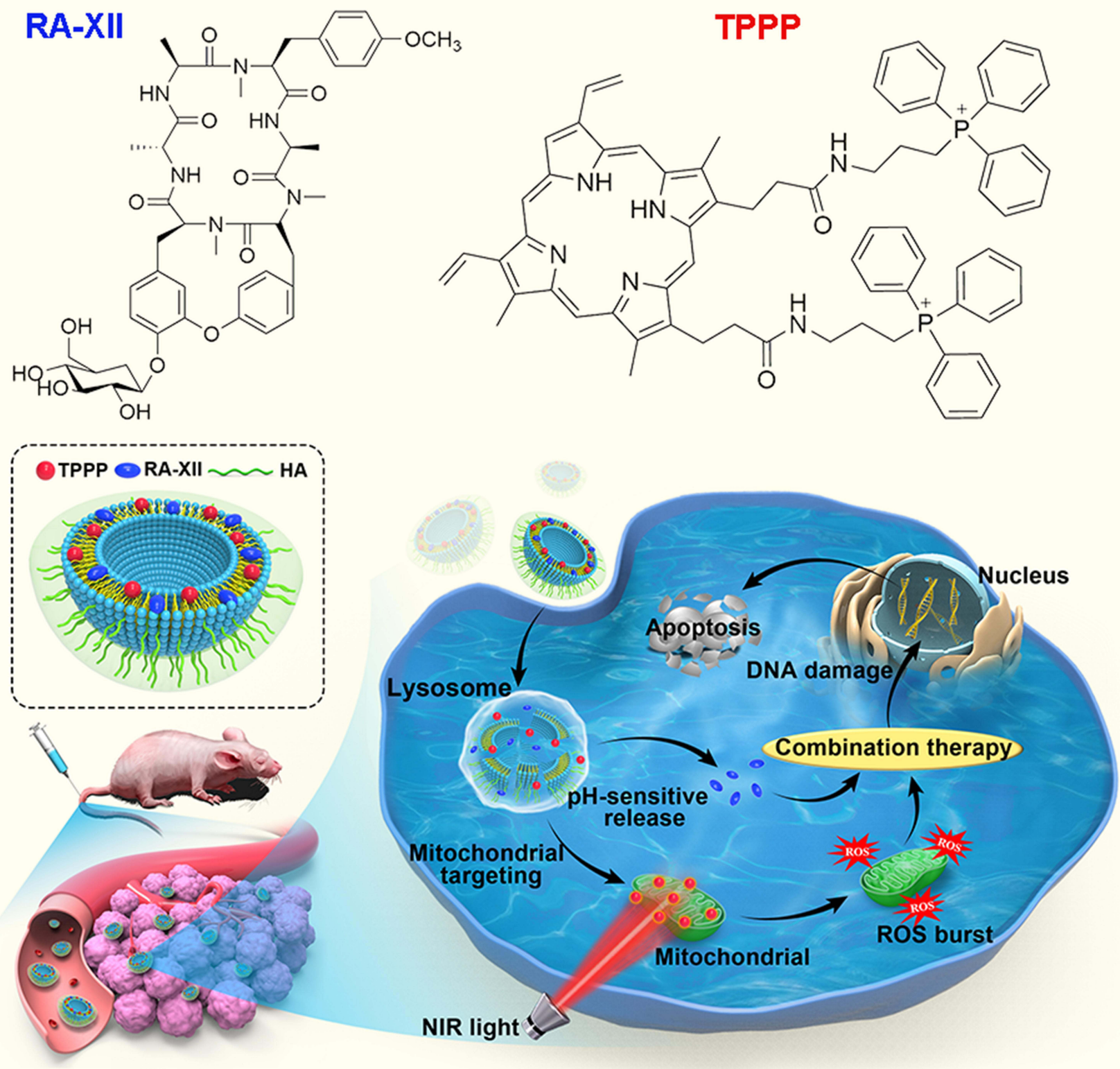

Scheme I Schematic illustration of the preparation of RA/TPPP-Lip and intracellular site-specific localized RA-XII and TPPP release for combination therapy after cell uptake of RA/TPPP-Lip with laser irradiation. RA-XII was served as a chemotherapy drug and TPPP was used for PDT under $660 \mathrm{~nm}$ irradiation.

(CHOL) and 1,2-Dioleoyl-sn-glycero-3-phosphoethanolamine (DOPE) were purchased from Avanti Polar Lipids (Alabaster, USA). 1.2-Dipalmitoyl-sn-glycero-3-phosphoethanolamine (DPPE) was purchased from Corden Pharma Switzerland LLC (Switzerland). DSPE-mPEG2000 was purchased from Shanghai YuanYe Bio-Technology Co., Ltd (Shanghai, China). Triphenylphosphine and 3-bromopropylamine hydrobromide were obtained from Alfa Aesar (USA). RA-XII was isolated from $R$. yunnanensis and identified by mass spectrometry (MS) and nuclear magnetic resonance (NMR) by us. Sodium hyaluronate (molecular weight:
$5.6 \mathrm{kDa}$ ) was purchased from Shandong Freda Biopharmaceutical Co., Ltd. (Shandong, China). 3,3'Dioctadecyloxacarbocyanineperchlorate (DiO) was obtained from Sigma-Aldrich (St Louis, MO, USA). MitoTracker Red, MitoTracker Green, Hoechst 33342, LysoTracker Red and Annexin V-FITC/PI cell apoptosis kit were purchased from Invitrogen (Carlsbad, CA, USA). Calcein-AM/PI Cell Viability/Cytotoxicity Assay Kit and ROS Assay Kit were purchased from Shanghai Biyuntian Biotechnology Co., LTD (Shanghai, China). Ultrapure water was prepared using a Millipore Simplicity System (Millipore, Bedford, USA). 


\section{Characterization}

Hydrodynamic diameter of the samples was measured by a Malvern Zetasizer Nano instrument (Malvern Company, UK). Transmission Electron Microscope (TEM) images were acquired on a Hitachi HT7700 Exalens microscope at $120 \mathrm{KV}$. Fluorescence images were obtained by a confocal laser scanning microscope (LSM700, Zeiss, Germany). Flow cytometric assay was captured by an Attune NxT flow cytometer (Thermo Fisher Scientific, USA). Animal fluorescence (in vivo and ex vivo) imaging was performed using 3D visible-light imaging system for small animals (IVIS Spectrum, PerkinElmer, USA).

\section{Preparation of RA/TPPP-Lip}

TPP-NH ${ }_{2}$ (3-aminopropyl) triphenylphosphonium was synthesized by the substitution of triphenylphosphine for bromine in 3-bromopropylamine. ${ }^{37}$ Then, the purified TPPP was synthesized via the amide coupling reaction between protoporphyrin-IX (-COOH) and TPP$\mathrm{NH}_{2}\left(-\mathrm{NH}_{2}\right) .{ }^{1} \mathrm{H}$ NMR, ${ }^{13} \mathrm{C}$ NMR and LC-MS methods were used to confirm the structure of TPPP. ${ }^{1} \mathrm{H}$ NMR (400 $\left.\mathrm{MHz}, \mathrm{CDCl}_{3}\right) \delta 10.14-9.50$ (overlapped, $3 \mathrm{H}, 64,65,68$ ), 8.68 (br.s, 1H, 78), 8.10 (br.s, 1H, 67), 7.85-6.56 (overlapped, 33H, 1, 2, 3, 4, 5, 7, 8, 9, 11, 12, 13, 14, 16, 17, 18, $23,24,25,26,27,29,30,31,33,34,35,36,38,39,40$, 69, 71, 83), 6.46-5.88 (overlapped, 5H, 66, 70, 72), 4.22 (br.s, 4H, 20, 42), 3.98-2.62 (overlapped, 19H, 22, 44, 73, 74, 75, 76, 79, 81), 2.17 (br.s, 2H, 80), 1.20 (overlapped, $8 \mathrm{H}, 21,43,56,86) ;{ }^{13} \mathrm{C}$ NMR (100 MHz, DMSO- $\left.d_{6}\right) \delta$ 172.35 (2C, 77, 82), 135.23 (3C, 51, 61, 71), 133.72 (21C, $2,4,8,12,13,17,23,24,27,30,31,33,35,39,45,46$, 54, 55, 57, 60, 62), $130.51(24 \mathrm{C}, 1,3,5,7,9,11,14,16$, $18,25,26,29,34,36,38,40,47,49,50,53,58,59,63$, 69), 121.45 (2C, 65, 68), 118.51 (7C, 6, 10, 15, 32, 37, 70, 72), 98.62-96.47 (3C, 28, 66, 67), 38.89 (4C, 22, 44, 76, 81), 22.45 (6C, 20, 21, 42, 43, 75, 80), 18.33 (2C, 73, 74), 13.04 (1C, 79), 11.74 (1C, 86).

RA/TPPP-Lip (without HA) composed of S100: CHOL: DPPE: DOPE: DSPE-mPEG2000: TPPP: RA-XII at the molar ratios of 4:1:1:2:0.4:0.5:0.4 were first prepared by thin-film hydration method. In a typical method, they were dissolved in chloroform-methanol, evaporated to dryness by a rotary evaporator under vacuum to obtain a thin lipid film. Then, the lipid film was hydrated in $\mathrm{pH} 7.4$ phosphate buffer saline (PBS) by rotating the round-bottom flask at $68{ }^{\circ} \mathrm{C}$ for $1 \mathrm{~h}$. The obtained multilamellar vesicles were sonicated with an ultrasonic probe for $8 \mathrm{~min}$ at $100 \mathrm{~W}$ with pulsing (pulse on $1 \mathrm{~s}$, off $1 \mathrm{~s}$ ) in an ice water bath, and then extruded through $0.22 \mu \mathrm{m}$ filter membranes.

To prepare the HA-conjugated liposomes (RA/TPPPLip), the surface modification of the resulting liposomes was prepared according to the literature ${ }^{38}$ with some modification. Briefly, sodium hyaluronate was hydrated in the 2-(N-morpholino) ethanesulfonic acid buffer (Mes buffer) for some time, preactivated through incubation with EDC and NHS for $1 \mathrm{~h}$ at $37{ }^{\circ} \mathrm{C}$, and then added into the liposome suspension. The next step was adjusting the $\mathrm{pH}$ to 7.8 by $100 \mathrm{mM}$ borate buffer and incubating overnight at $37{ }^{\circ} \mathrm{C}$. After incubation, HA modified liposomes (RA/ TPPP-Lip) were separated by dialysis bag to remove the excess reagents. Additionally, the Empty Liposome, RALip, RA-Lip (without HA) and TPPP-Lip were prepared by using the similar procedures.

\section{Characterization of RA/TPPP-Lip}

The size and morphology of the RA/TPPP-Lip were observed by using TEM. Particle size distribution and zeta potential were performed on Malvern Zetasizer nano instrument (Malvern, UK). The encapsulation efficiency (EE) of the liposomes was determined as described below. In brief, after destruction of RA/TPPP-Lip with Triton X-100, liquid chromatography-tandem mass spectrometry (LC-MS/MS) was used to determine the concentrations of RA-XII. EE was calculated using the following equation:

$\mathrm{EE}(\%)=($ the amount of loaded RA-XII)/(the amount of added RA-XII) $\times 100 \%$

In vitro drug release was conducted using a dialysis method. One milliliter of RA/TPPP-Lip was placed in dialyzed bag (MWCO 14000) in $50 \mathrm{~mL}$ PBS buffer and stirred at $100 \mathrm{rpm}$ at $37{ }^{\circ} \mathrm{C}$. Aliquots $(200 \mu \mathrm{L})$ of the release medium were withdrawn for analysis and replaced with fresh medium at different time points (up to $24 \mathrm{~h}$ ). The amount of RA-XII released from RA/TPPP-Lip was measured by LC-MS/MS. The percentage of RA-V released at different time points was calculated using the equation:

Release $(\%)=$ cumulative amount released/total amount in liposomes $\times 100 \%$

\section{Cell Culture and Confocal Fluorescence Imaging}

Human colorectal cancer cell line HCT116 and human normal intestinal epithelial cell line NCM460 were 
obtained from Type Culture Collection of Chinese Academy of Sciences (Shanghai, China). HCT116 and NCM460 cells were cultured at $37{ }^{\circ} \mathrm{C}, 5 \% \mathrm{CO}_{2}$. One day before imaging, HCT116 and NCM460 cells were seeded in 96-well flat-bottom culture plates for $24 \mathrm{~h}$. Confocal fluorescence imaging was performed using a ZEISS Laser Scanning Microscope (LSM700, Zeiss, Germany) with a $63 \times$ oil-immersion objective.

\section{In vitro Cytotoxicity Assay}

For cytotoxicity assay of RA/TPPP-Lip, the seeded HCT116 cells were cultured in 96-well plates overnight and then treated with different concentrations of Empty Liposome, Free TPPP, Free RA-XII, RA-Lip, RA-Lip (without HA) and RA/TPPP-Lip for $24 \mathrm{~h}$ at $37{ }^{\circ} \mathrm{C}$. The dose of Free RA-XII and TPPP was equal to the amount of RA-XII and TPPP in RA/TPPP-Lip. In addition, in order to explore the cytotoxicity after laser irradiation, the HCT 116 cells were irradiated with $660 \mathrm{~nm}$ laser for 5 minutes $\left(1 \mathrm{~W} / \mathrm{cm}^{2}\right)$, and then incubated in the dark for 18 hours. Cell viability was determined by SRB assay. Furthermore, in order to assess the therapeutic effect of RA/TPPP-Lip in vitro, the seeded HCT116 cells were irradiated with $660 \mathrm{~nm}$ laser and further incubating for $18 \mathrm{~h}$, then each group of cells was stained with Calcein$\mathrm{AM}$ and PI for confocal fluorescence imaging.

\section{Confocal Imaging of Intracellular ROS}

After RA/TPPP-Lip treatment, DCFH-DA was employed as a ROS-responsive probe $(10 \mu \mathrm{mol} / \mathrm{L})$ to evaluate the intracellular ROS level. After seeding overnight, the HCT116 cells were incubated with RA/TPPP-Lip for $6 \mathrm{~h}$, followed by $660 \mathrm{~nm}$ laser irradiation $\left(1 \mathrm{~W} / \mathrm{cm}^{2}\right)$. Immediately, the cells were washed with PBS three times and then stained with DCFH-DA before imaging by LSM $700 .^{39}$

\section{Flow Cytometry of Annexin V-FITC and PI}

For apoptosis assays, the HCT116 cells were harvested following treated and double-stained with Annexin V and PI immediately before analysis by flow cytometry (at least 50,000 cells for each experiment).

\section{In vivo Therapy}

Specific pathogen-free (SPF) female BALB/c nude mice, 6-8 weeks of ages, were used for in vivo therapy.
A subcutaneous-tumor-bearing nude mouse model was established by subcutaneously inoculating with $200 \mu \mathrm{L}$ of PBS containing $1 \times 10^{6}$ HCT116 cells into the arm pit of the right anterior limb. All animal procedures were carried out accordance with the Guidelines for Care and Use of Laboratory Animals of China Pharmaceutical University and were approved by the Animal Care Committee of China Pharmaceutical University. When the tumor volume grew up to $100 \mathrm{~mm}^{3}$, each group of nude mice was intravenously injected with $200 \mu \mathrm{L}$ of different formulations every three days for 12 days. Six groups were: (1) PBS (control), (2) Laser, (3) TPPP-Lip with Laser, (4) RA-Lip, (5) RA/TPPP-Lip, (6) RA/TPPPLip with Laser. The mice of group (2), group (3) and group (6) were irradiated with $660 \mathrm{~nm}$ laser for $5 \mathrm{~min}$ respectively at $24 \mathrm{~h}$ post-intravenous injection. In the administration group, the relative dose of RA-XII was about $20 \mathrm{mg} / \mathrm{kg}$, and the relative dose of TPPP was about $5 \mathrm{mg} / \mathrm{kg}$, with the same concentration of phospholipids. Body weight and tumor size of mice were recorded three times a week. After 12 days, mice were euthanized, and tumors along with normal organs were harvested and then fixed in $4 \%$ formaldehyde for $H \& E$ staining and TUNEL analysis.

\section{In vivo Imaging Study}

The HCT116 cells $\left(1 \times 10^{6}\right.$ cells) were subcutaneously (s.c.) implanted on the 7-week female BALB/c nude mice. When the tumors were ready for use, RA/TPPP-Lip and RA/TPPPLip (without HA) were injected through the tail vein at RAXII dosage of $10 \mathrm{mg} / \mathrm{kg}$. At 2, 8, and $24 \mathrm{~h}$, the mice were anesthetized and obtained the fluorescence images using an IVIS spectrum instrument. At $24 \mathrm{~h}$ post injection of RA/ TPPP-Lip and RA/TPPP-Lip (without HA), the mice were euthanized, and then the tumor, heart, liver, lungs, kidneys and spleen were taken for ex-vivo imaging.

\section{Results and Discussion Characterization of RA/TPPP-Lip}

Protoporphyrin-IX (-COOH) and TPP- $\mathrm{NH}_{2}\left(-\mathrm{NH}_{2}\right)$ were conjugated by the amide bond. Modification of protoporphyrin-IX with mitochondrial target functional group TPP to obtain TPPP (Figure S1, Supporting Information), and the successful synthesis of TPPP was verified by ${ }^{1} \mathrm{H}$ NMR, ${ }^{13} \mathrm{C}$ NMR and LC-MS analyses, separately (Figure S2, Supporting Information). Figure 1 summarizes main physical-chemical properties of RA/TPPP-Lip. The 
morphology of RA/TPPP-Lip was observed by transmission electron microscopy (TEM). As Figure 1A showed, RA/TPPP-Lip displayed spherical shape with a clear coreshell structure and uniform diameters. The average particle size of the targeted RA/TPPP-Lip determined with a Malvern Zetasizer nano instrument were $184.4 \pm 6.6$ $\mathrm{nm}$ in diameter with a polydispersity index (PDI) less than 0.3 , and were $157.8 \pm 9.6 \mathrm{~nm}$ before HA-modification (Figure 1B). The Zeta potential of the obtained RA/TPPPLip was changing from $-41.3 \pm 1.4 \mathrm{mV}$ to $-56.32 \pm 1.9$ $\mathrm{mV}$ after modification with HA, confirming successful conjugation of HA. The encapsulation efficiency of RAXII in liposome was about $71 \%$ with liquid chromatography-tandem mass spectrometry (LC-MS/MS) analysis. Then, the release behavior of RA-XII under different $\mathrm{pH}$ conditions was investigated (Figure 1C). After 24 hours of incubation, RA-XII release from RA/TPPP-Lip reached a maximum approximately $80 \%$ at $\mathrm{pH} 5.0$. However, the release of RA-XII obviously slowed up at $\mathrm{pH} 6.0$ and smaller amount of RA-XII was released at $\mathrm{pH}$ 7.4. As lysosomes are organelle with acidic environment $(\mathrm{pH} 5-$
6), RA/TPPP-Lip was found to be sensitive to the acidic condition for the targeted of drug release in the lysosomes of the colon cancer cells. In addition, the stability of RA/ TPPP-Lip was investigated. As shown in Figure S3, with the increase of storage days, the hydrodynamic diameter of RA/TPPP-Lip was still maintained at about $170 \mathrm{~nm}$, and there was no sedimentation observed by naked eyes, and it has good stability in the solution. In a word, the combined nano therapy system RA/TPPP-Lip was successfully constructed to deliver RA-XII and TPPP at the same time, which could precisely trigger site-specific release in response to the tumor acidic microenvironment.

\section{Cellular Selectivity}

3,3'-Dioctadecyloxacarbocyanineperchlorate (DiO) was doped into the liposome shells as a fluorescence tracker, in order to test targeting specificity of RA/TPPP-Lip in colon cancer cells. RA/TPPP-Lip at $50 \mu \mathrm{g} / \mathrm{mL}$ were incubated with CD44 receptors overexpressed HCT116 cells. As expected, the fluorescence of HCT116 cells steadily increased with the time of incubation and got to the
A

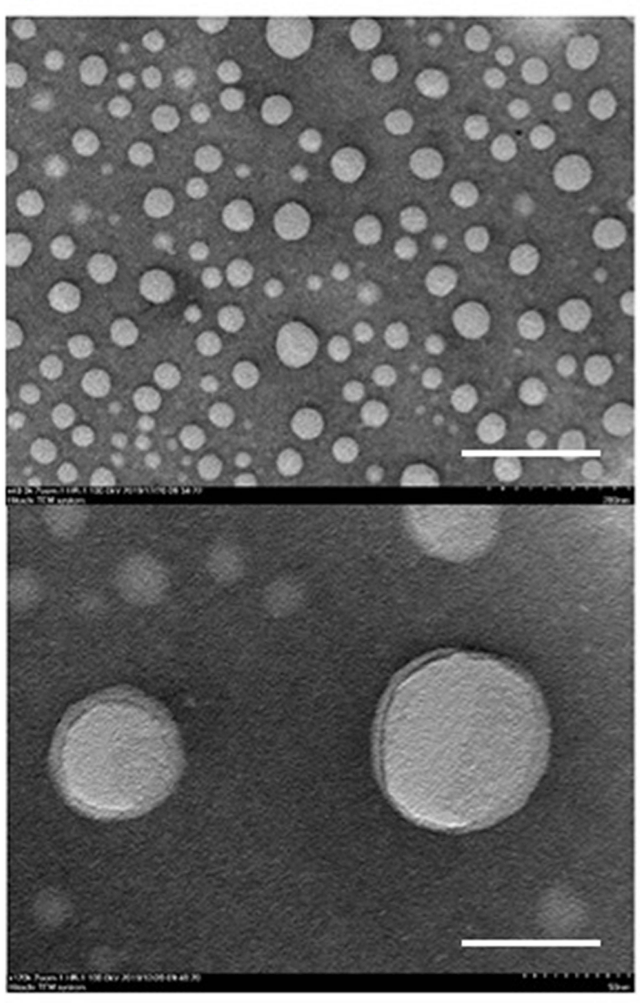

B

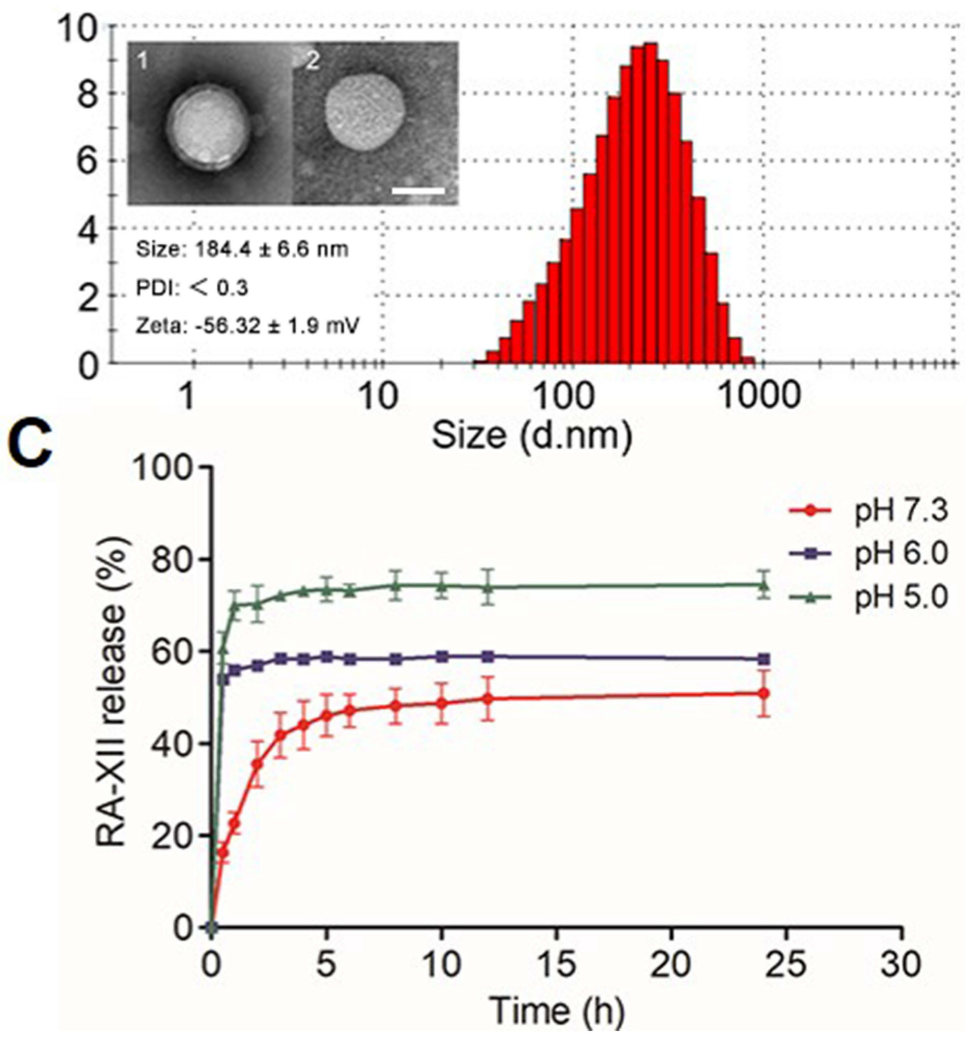

Figure I Characterizations of RA/TPPP-Lip. (A) Transmission electron microscopy image of RA/TPPP-Lip. Scale bar: $200 \mathrm{~nm}$, $500 \mathrm{~nm}$. (B) The particle size distribution of RA/TPPP-Lip. Inset: TEM image of RA/TPPP-Lip (I) and RA/TPPP-Lip (without HA) (2); Particle size, PDI, and zeta potential of RA/TPPP-Lip. Scale bars: I00 nm. (C) Schematic diagram of in vitro release of RA-XII from RA/TPPP-Lip at $\mathrm{pH} 7.3,6.0$ and 5.0. Data are presented as mean \pm SD $(n=3)$. 
maximum fluorescence intensity at $8 \mathrm{~h}$ (Figure 2A), demonstrating that RA/TPPP-Lip were efficiently uptake by HCT116 cells. RA/TPPP-Lip was also incubated with NCM460 cells, which are normal colon epithelial cells expressing low levels of CD44 receptors. Compared to HCT116 cells, only a tiny amount of fluorescence was shown in the NCM460 cells. The significant differences in fluorescence between the two cells demonstrated that the well targeting specificity of RA/TPPP-Lip towards HA integrin-rich colon cancer cells. To further test that the targeting specificity of RA/TPPP-Lip was owed to the selective recognition of $\mathrm{HA}$ to $\mathrm{CD} 44$ receptors on colon cancer cells, RA/TPPP-Lip without HA modification and RA/TPPP-Lip were monitored cellular uptake in HCT116 cells. In Figure 2B, the HCT116 cells displayed strong

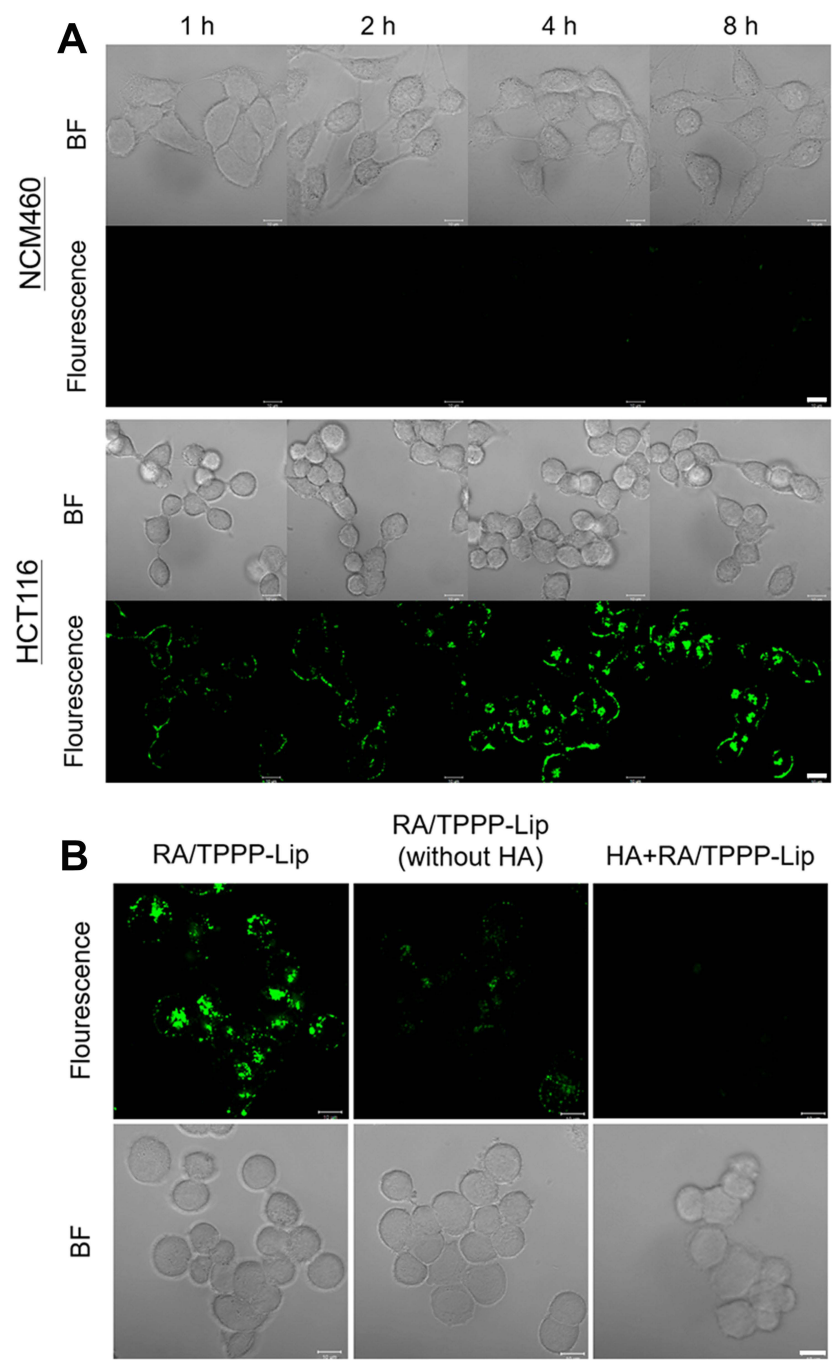

Figure 2 (A) Real-time confocal fluorescence images display the uptake of RA TPPP-Lip by HCTII 6 and NCM460 cells. Scale bars: $10 \mu \mathrm{m}$. (B) Confocal fluorescence images of HCTII6 cells incubated with RA/TPPP-Lip, RA/TPPP-Lip (without $\mathrm{HA}$ ) or HA+RA/TPPP-Lip for $4 \mathrm{~h}$. Scale bars: $10 \mu \mathrm{m}$. fluorescence after $4 \mathrm{~h}$ incubation of RA/TPPP-Lip, conversely, the cells incubated with RA/TPPP-Lip without HA modification showed little fluorescence intensity under the similar experimental condition. Furthermore, when pretreated with excessive free HA, there was almost no fluorescence in the cells because the internalization of RA/TPPP-Lip was blocked. The results suggested that RA/ TPPP-Lip achieved the targeting capabilities to HCT116 cells, which was by means of the particular interaction of HA with CD44 receptors and HA-mediated endocytosis.

\section{Colocalization Assay}

For investigating the intracellular location of RA/TPPPLip, HCT116 cells incubated with RA/TPPP-Lip were costained with Hoechst 33342, Mito Tracker Red and LysoTracker Red, respectively. As observed in Figure 3A, the fluorescence of RA/TPPP-Lip in the cell almost completely coincided with LysoTracker Red, but did not match in the case of Hoechst 33342 and MitoTracker Red. Consequently, these results suggested that RA/TPPP-Lip entered CD44 receptors-rich colon cancer cells by receptor-mediated endocytosis and were transported to the lysosomes. In addition, as shown in Figure 3B, HCT116 cells were pretreated with TPPP for $12 \mathrm{~h}$, the green fluorescence emitted by MitoTracker Green was well overlapped with red fluorescence of TPPP, indicating the cooperative targeting and localization of TPPP in mitochondria.

\section{RA/TPPP-Lip in vitro Therapy Studies}

In our study, RA-XII and TPPP were incorporated into RA/TPPP-Lip to build a synergistic nanosystem for highly efficient chemotherapy and PDT combination therapy in colon cancer.

The in vitro cytotoxic activity experiments of RA/ TPPP-Lip were assessed by the SRB assay. Firstly, as Figure 4A showed, empty liposome exhibited negligible cytotoxicity, which suggested the biocompatibility of liposome for drugs delivery. Moreover, compared with free TPPP without cytotoxic effect, in vitro cytotoxicity of TPPP-Lip combined with laser on HCT116 cells was significantly enhanced. After coating RA-XII in liposomes, its cytotoxicity increased to a certain extent, especially at low drug concentrations. More significantly, after RA-XII and TPPP were co-loading into the targeting carrier, anti-tumor activity of the resulting co-delivery system is significantly enhanced under laser irradiation. Subsequently, the therapeutic 


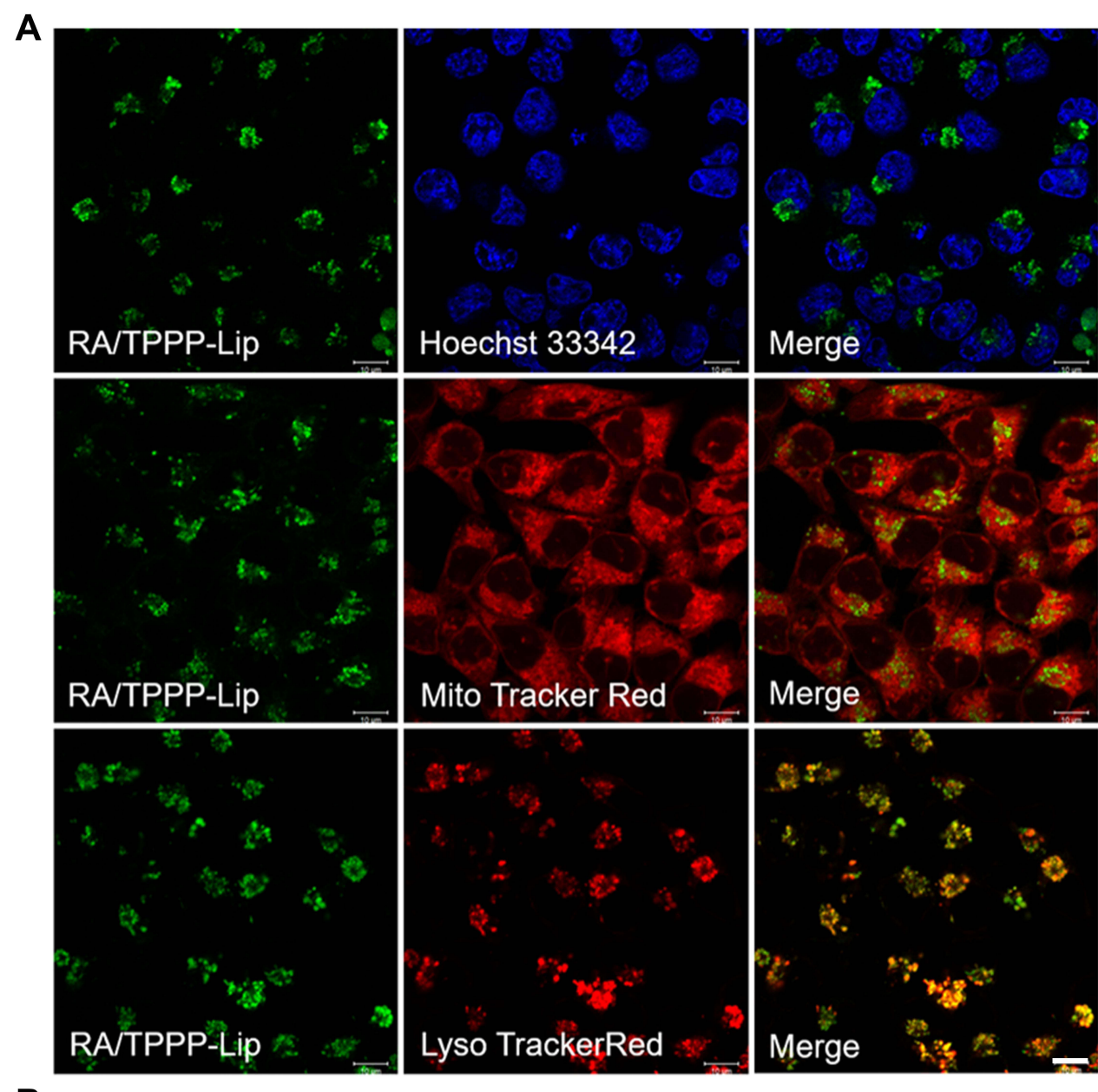

B

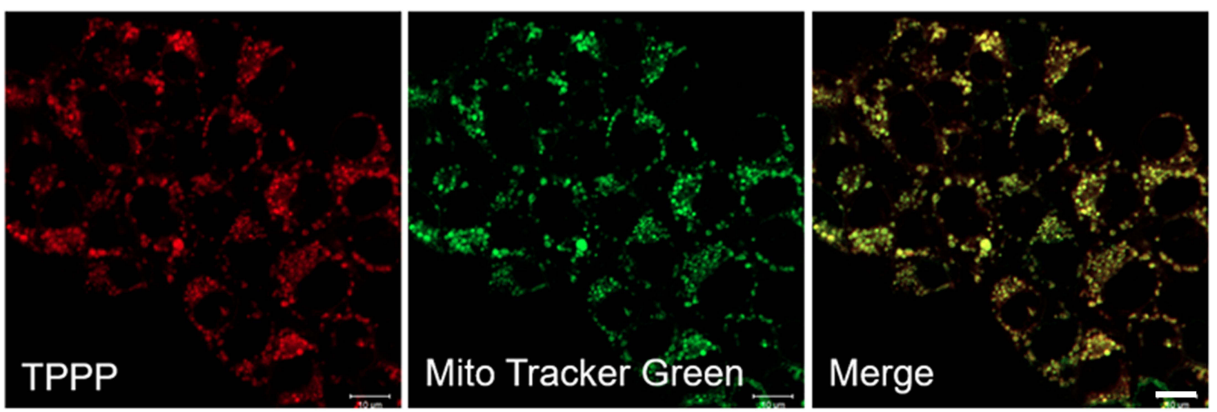

Figure 3 (A) Colocalization images of RA/TPPP-Lip in HCTII6 cells. Cells were incubated with RA/TPPP-Lip for $4 \mathrm{~h}$ and then incubated with I00 nM Hoechst 33342 , MitoTracker Red, or LysoTracker Red for 10 min. (B) Confocal images for analyzing mitochondria co-localization of TPPP in HCTII6 cell lines. Scale bars: $10 \mu \mathrm{m}$.

effect of RA/TPPP-Lip was evaluated after exposure to laser. In Figure 4B, after incubating HCT116 cells with different concentrations of RA/TPPP-Lip, cell viability decreased in a concentration-dependent manner after $660 \mathrm{~nm}$ laser irradiation. For further confirming the results, live/dead staining was used to evaluate living/ dead HCT116 cells after different treatments, which respectively showed green fluorescence for living cells and red fluorescence for dead cells in the confocal imaging system. It was easily shown that the lethality of HCT116 cells with RA/TPPP-Lip treatment plus laser irradiation was at a significantly higher rate than without laser irradiation (Figure 4C). The above cytotoxicity evaluations all confirmed that compared with using RA-Lip alone for chemotherapy or using TPPPLip alone for photodynamic therapy, the combination system RA/TPPP-Lip could significantly improve colon cancer treatment effect. 
A

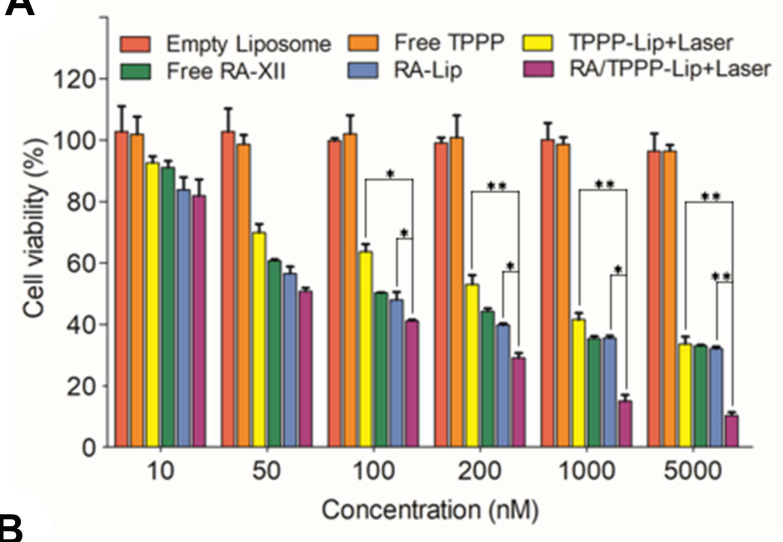

B

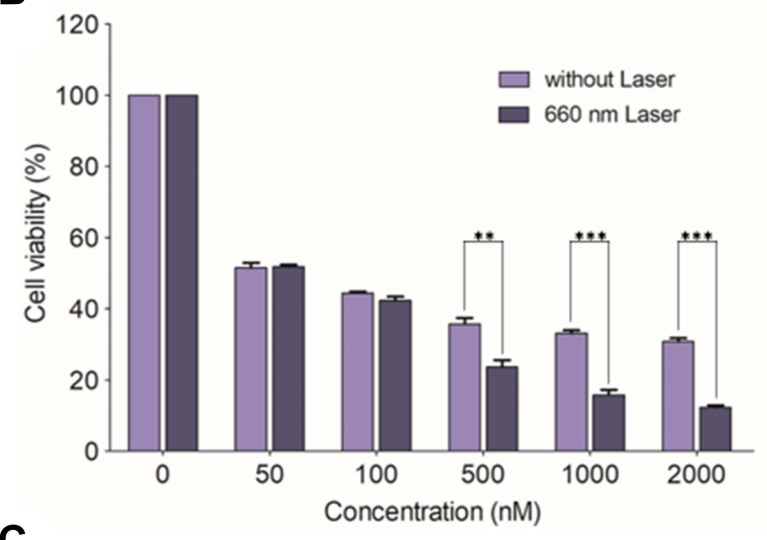

C

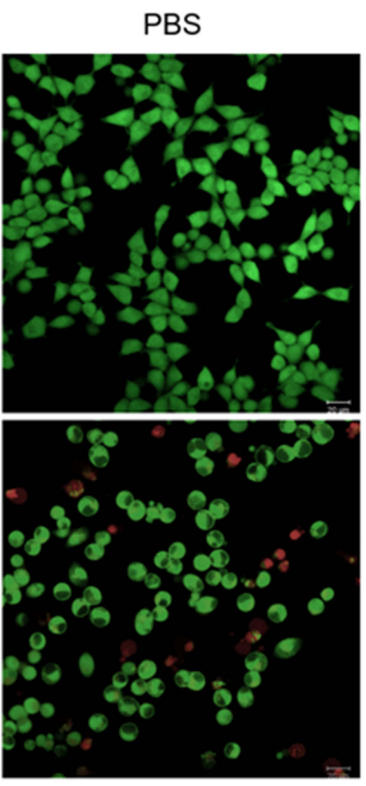

TPPP-Lip+Laser

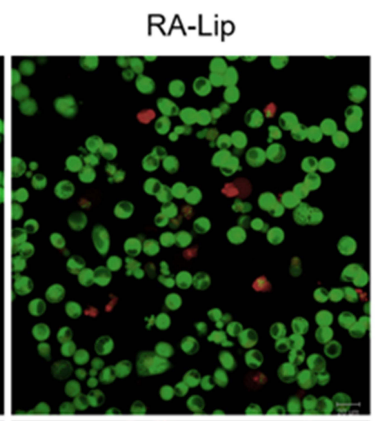

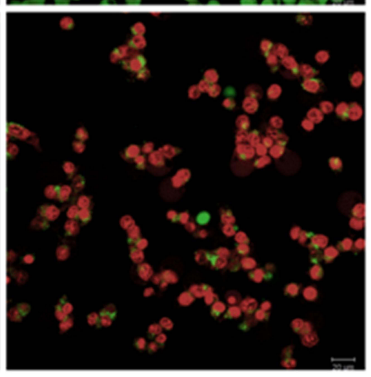

RA/TPPP-Lip+Laser
Figure 4 In vitro therapeutic study of RA/TPPP-Lip. (A) Relative cell viabilities of HCTII 6 cells treated with Empty Liposome, Free TPPP, TPPP-Lip+Laser, Free RAXII, RA-Lip, and RA/TPPP-Lip+Laser for 24h. $* P<0.05$, **P $<0.01$. (B) Relative cell viabilities of HCTII6 cells treated with different concentrations of RA/TPPP-Lip without or with $660 \mathrm{~nm}$ laser irradiation. **P $<0.01$, ***P $<0.001$. (C) Representative images of HCTII6 cells co-stained by Calcein-AM (green) and PI (red) after treatment with PBS (control), RA-Lip, TPPP-Lip with $660 \mathrm{~nm}$ laser irradiation, and RA/TPPP-Lip with $660 \mathrm{~nm}$ laser irradiation. Data represent mean \pm s.d. $(n=3)$. Scale bar: $20 \mu \mathrm{m}$.

\section{ROS Generation and Combinational} Therapeutic Effect on Colon Cancer Cells

To investigate the mechanisms of TPPP mediated antitumor activity, we performed a series of in vitro experiments. The impact of RA/TPPP-Lip and laser irradiation treatments on oxidative stress in HCT116 cells was explored. Intracellular ROS level was analyzed by DCFH-DA staining. As shown in Figure 5A, cells incubated with TPPP-Lip and RA/TPPP-Lip all exhibited strong green fluorescence after laser irradiation treatment, while the other three groups displayed no obvious fluorescence, demonstrating that TPPP in RA/TPPP-Lip worked following laser irradiation, so that the intracellular ROS level increased significantly.

The phospholipid-binding protein Annexin-V exhibits a high affinity towards inverted phosphatidylserine at an early stage of apoptosis. And PI, a nucleic acid dye, can pass through the damaged cell membrane and stain with nucleus as the cells are in late apoptotic or dead. Therefore, cells were classified with Annexin V/PI staining as viable cells (Annexin V-/PI-), early apoptotic cells (Annexin $\mathrm{V}+/ \mathrm{PI}-)$, late apoptotic cells (Annexin $\mathrm{V}+/ \mathrm{PI}+$ ) and necrotic cells (Annexin V-/PI+).

To further study the therapy effect of RA/TPPP-Lip, flow cytometry experiments were carried out to distinguish between live and dead cells at different stages. After incubated with $20 \mu \mathrm{g} / \mathrm{mL} \mathrm{RA}$ /TPPP-Lip for $6 \mathrm{~h}$ and then irradiated with a $660 \mathrm{~nm}$ laser immediately, the cells were stained with Annexin V-FITC and PI. In Figure 5B, compared with other groups, the proportion of apoptosis cells in RA/TPPP-Lip and laser irradiation groups increased (30\%), and the proportion of live cells decreased (29.3\%). The population of apoptotic cells in RA/TPPP-Lip group was similar to that in RA-Lip group and TPPP-Lip+Laser group. At the same time, the degree of apoptosis of HCT116 cells treated with RA/TPPP-Lip and laser was much higher than that in other groups. Taken together, all the results indicated that the nano therapeutic platform RA/ TPPP-Lip greatly improved the killing efficiency of colon cancer cells through the combination of chemotherapy and photodynamic therapy.

\section{In vivo Targeted Imaging of Subcutaneous Tumor-Bearing Mice}

We examined the targeting capability of RA/TPPP-Lip in HCT116 tumor-bearing BALB/c nude mice. After injection 


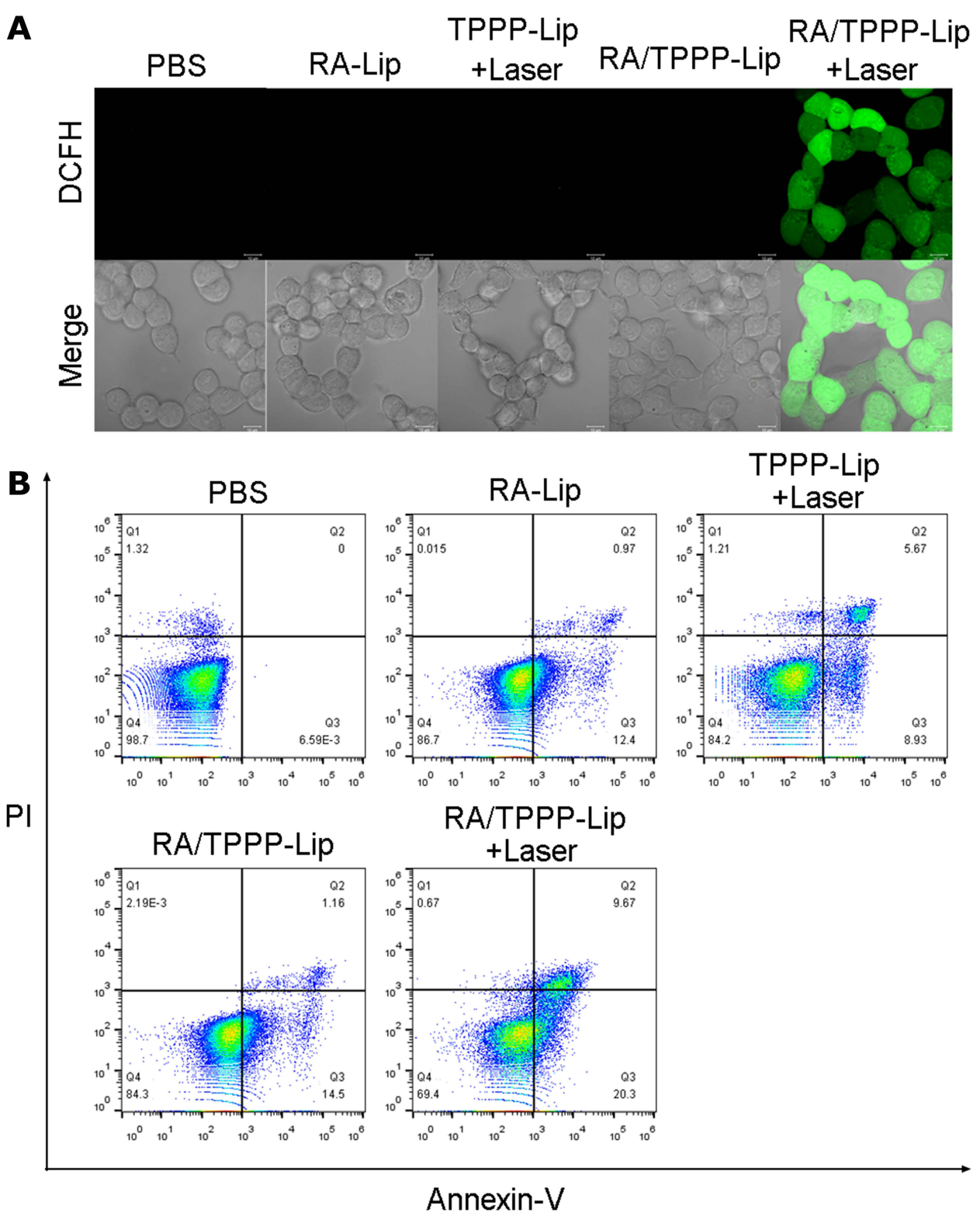

Figure 5 (A) Confocal microscopy image of ROS levels in HCTI I6 cells treated with PBS (control), RA -Lip, TPPP-Lip with 660 nm laser irradiation, RA/TPPP-Lip, and RA/ TPPP-Lip with $660 \mathrm{~nm}$ laser irradiation. Scale bar: $10 \mu \mathrm{m}$. (B) Flow cytometry assays of HCTII6 cells treated with PBS (control), RA-Lip, TPPP-Lip with $660 \mathrm{~nm}$ laser irradiation, RA/TPPP-Lip, and RA/TPPP-Lip with $660 \mathrm{~nm}$ laser irradiation.

of RA/TPPP-Lip into the tail vein of HCT116 tumor-bearing mice, the fluorescence in tumor region became stronger than the surrounding normal tissues as time increased, and still maintained strong signals at $24 \mathrm{~h}$ (Figure 6), revealing the effective tumor accumulation of RA/TPPP-Lip. The tumor tissue showed bright fluorescence while other organs exhibited nearly no fluorescence as shown in the ex vivo fluorescence images. In contrast, the fluorescence signals almost distributed in different organs and tumor tissues after intravenous injection with RA/TPPP-Lip (without HA), and hardly accumulated in the tumor tissue relative to other organs at
$24 \mathrm{~h}$ post-injection, proving that HA plays a key role in the targeting drug-delivery to colon tumor. These results were sufficient to prove that RA/TPPP-Lip can effectively deliver RA-XII and TPPP to tumor sites through the active targeting ability mediated by surface-modified HA.

\section{In vivo Tumor Treatment Research of RA/ TPPP-Lip}

In vivo anti-tumor effect of RA/TPPP-Lip was verified by experiments using mice. Nude mice-bearing HCT116 tumor were randomized into six groups and treated as 
A

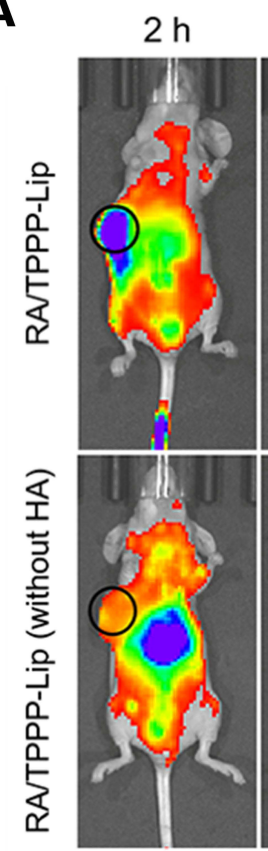

$8 \mathrm{~h}$

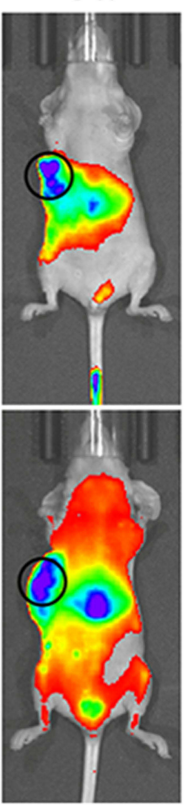

$24 \mathrm{~h}$

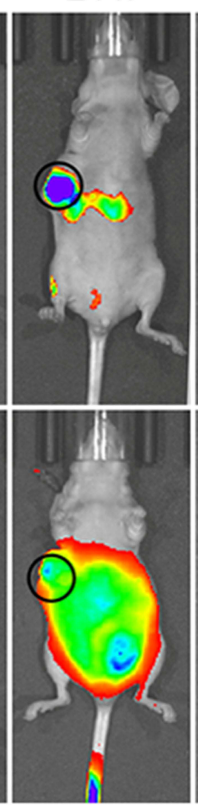

\section{Ex vivo}

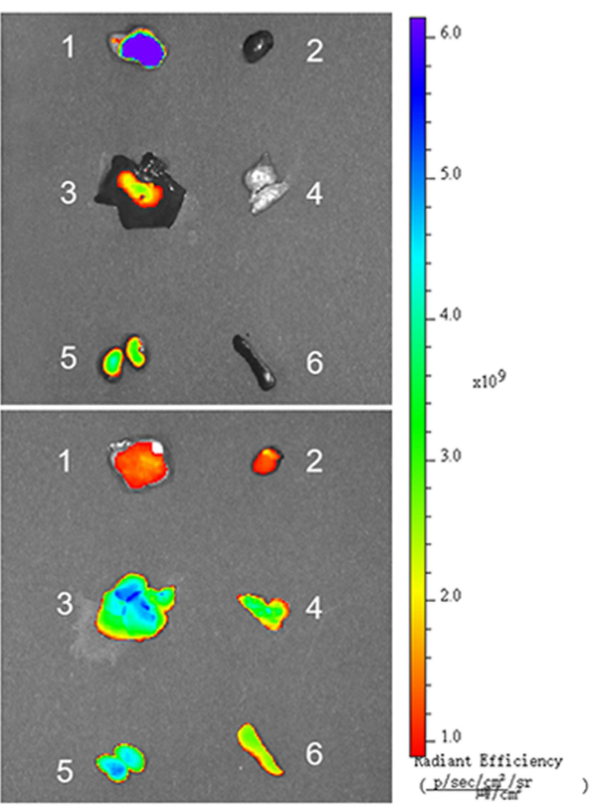

B

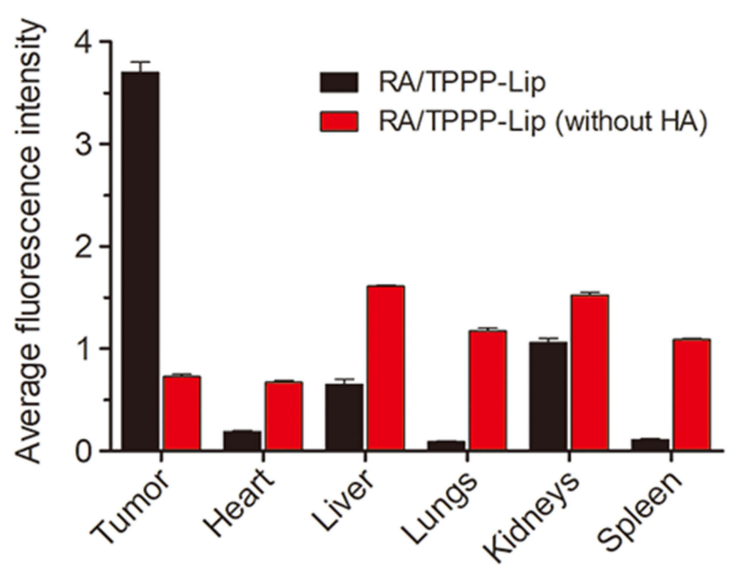

Figure 6 (A) Time-dependent fluorescence images in vivo and ex vivo (I: tumor; 2: heart; 3: liver; 4: lungs; 5: kidneys; 6: spleen) of subcutaneous HCTI I6 tumor-bearing mice after tail vein injection of RA/TPPP-Lip or RA/TPPP-Lip (without HA). The sequence of red, orange, yellow, green, and blue corresponds to the increase in fluorescence intensity. (B) Ex vivo fluorescence intensity of tumor tissues and the major organs of nude mice at 24 post injection of RA/TPPP-Lip and RA/TPPP-Lip (without HA).

described: (1) PBS (control), (2) Laser, (3) TPPP-Lip +Laser, (4) RA-Lip, (5) RA/TPPP-Lip, (6) RA/TPPP-Lip +Laser. As shown in Figure 7A, the tumor volumes of mice in the PBS and Laser groups showed rapid growth, which suggested that there was little effect on the tumor growth of the mice treated with only laser light. The tumor growth of mice in the TPPP-Lip+Laser and RALip group show some degree of inhibition, which can be attributed to the toxicity of RA-XII and the laser lighttriggered toxicity of TPPP. Furthermore, the tumor growth trend in the RA/TPPP-Lip group without laser irradiation was close to that in the RA-Lip group. In contrast, the combined treatment with laser irradiation and RA/TPPPLip significantly inhibited the tumor growth in mice. The tumor volume inhibition rate exceeded 50\% after four treatments. After treatment, the inhibition of tumor weight was consistent with the result of tumor volume change trend (Figure 7B and D). And no obvious fluctuation was observed in the weight of mice for each group during the treatment period, demonstrating no evident side effects with these formulations (Figure 7C). In addition, the in vivo biological safety of RA/TPPP-Lip was further evaluated by H\&E staining with the organ tissues (eg, heart, liver, lungs, kidneys and spleen) of mice. No 
A

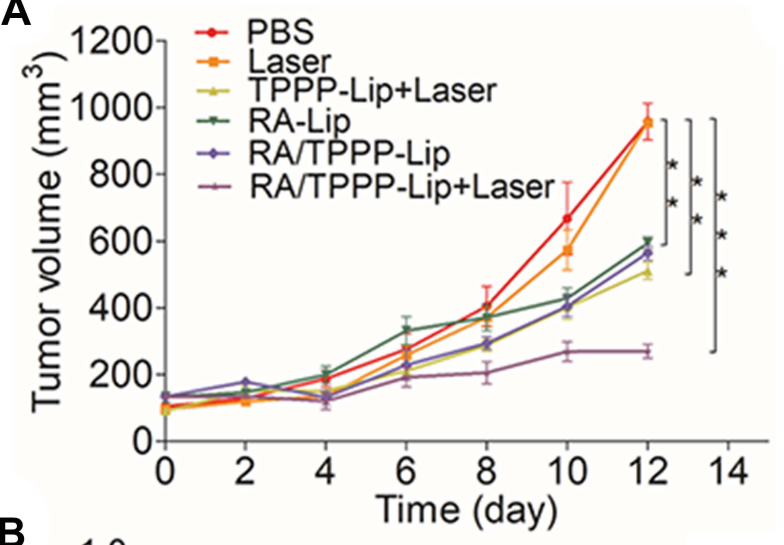

B
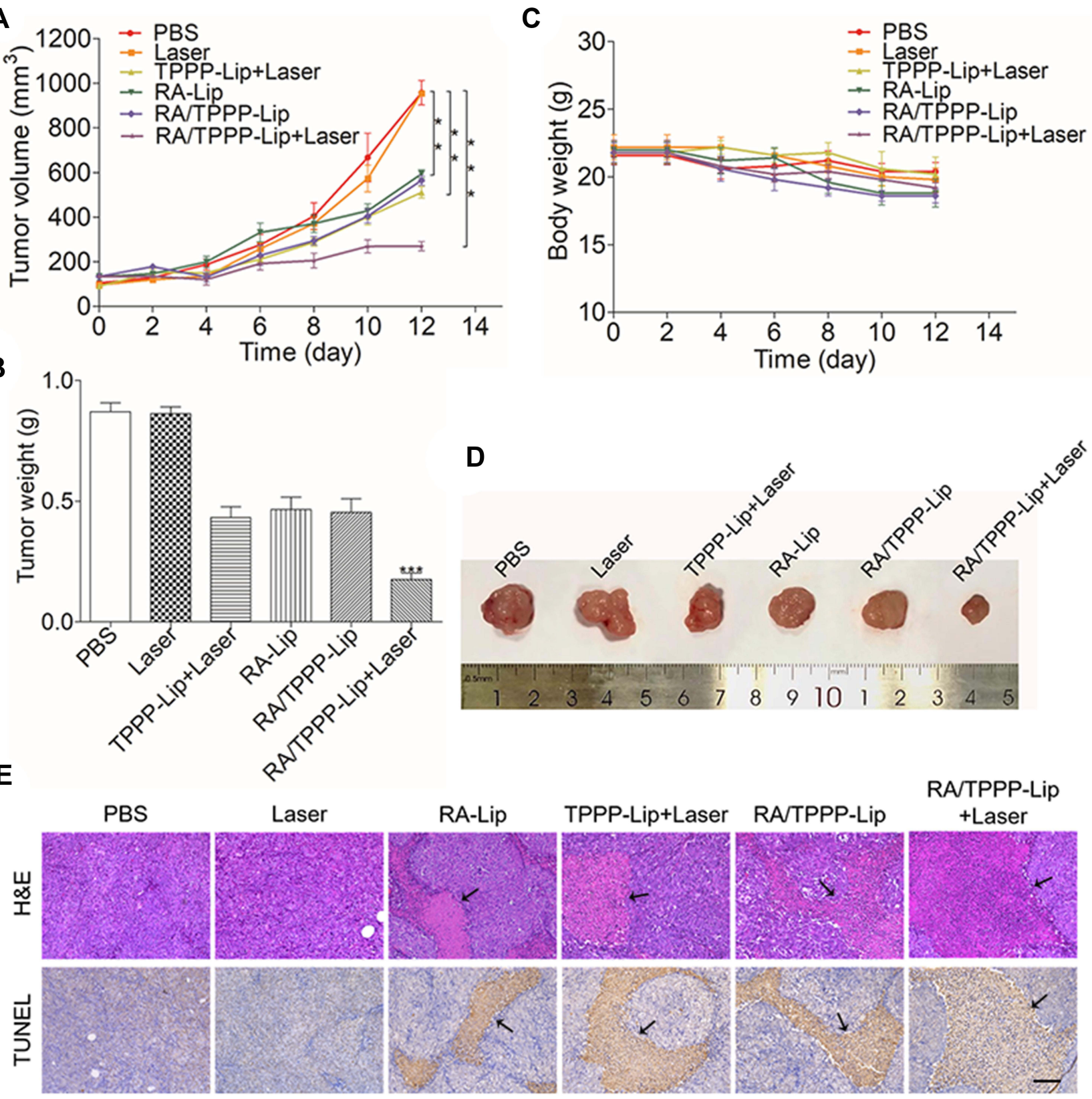

Figure 7 (A) Relative tumor volume $\left(\mathrm{V} / \mathrm{V}_{0}\right)$ changes. (B) Tumor weight of mice. (C) Changes in body weight of mice in different groups during treatment. (D) Representative images of HCTII6 tumors with different treatment at the end point of the experiment. (E) H\&E and TUNEL staining results of tumor tissues after treatment from different groups of mice. The arrows point to the area of cell necrosis. Scale bar: $100 \mu m$. Data represent mean \pm s.d. $(n=6$ mice per group). $* * P<0.01$, $* * * \mathrm{P}<0.001$.

apparent morphological alterations were observed in the tissues of the RA/TPPP-Lip-treated mice, relative to the age-matched healthy mice without treatment (Figure S4). These results showed that RA/TPPP-Lip was potent to kill colon cancer cells effectively without damage to normal cells.

In order to further evaluate the treatment efficacy on tumors, the mice were sacrificed and tumor tissues were harvested followed by histological analyses. The H\&E staining assays results showed that the tumor of PBS and Laser groups had no distinct injury and the necrosis effect were more evident in the group treated by RA/TPPP-Lip with laser than the RA-Lip and TPPP-Lip+Laser groups (Figure 7E). Moreover, TUNEL staining assay also demonstrated that the RA/TPPP-Lip combined with laser group had high levels of apoptosis, suggesting that the laser-responsive therapy based on RA/TPPP-Lip could activate apoptosis of tumor tissue. Taken together, the 
high combination of RA-XII and TPPP through nano platform shows great biocompatibility and excellent antitumor effects by inducing apoptosis of the colon cancer cells.

\section{Conclusion}

In conclusion, a therapy platform (RA/TPPP-Lip) based on the combination of chemotherapy and photodynamic therapy for highly efficient antitumor therapy was developed. Benefiting from the modification with HA on the liposomal shell and mitochondria-targeting photosensitizer wrapped in a bilayer, RA/TPPP-Lip achieved a highly selective targeted drug delivery for superb dispersibility, less cytotoxicity, good bioavailability, high hematology safety and in vivo biocompatibility. The $\mathrm{pH}$ response characteristic can precisely trigger the coordinated release of RA-XII and TPPP, which improves the efficiency of combined treatment. Moreover, the TPPP, we developed, could facilitate the accumulation of PS in mitochondria, thereby further improving the efficacy of PDT under laser light irradiation, and then induce cell apoptosis cooperated with RA-XII. We believe that TPPP, as an organelle-targeted photosensitizer molecule with good therapeutic effects, will be widely used in therapeutics. This organelle-specific PDT system provides an excellent opportunity to combat cancer or other mitochondria-associated diseases. In addition, the mitochondrial-targeted photosensitizer TPPP can also be loaded on functional liposomes together with other therapeutic reagents used for multimode treatment taking advantage of the synergistic therapeutic effects of the two therapies. Therefore, RA/TPPPLip is a promising platform for the controlled release of multiple drugs with controlled release rates at desired sites, which could benefit the treatment of colon cancer.

\section{Acknowledgments}

The authors wish to express sincere gratitude to the National Natural Science Foundation of China (21602255, 32070356), the National New Drug Innovation Major Project (2017ZX09309027) of Ministry of Science and Technology of China, the "Double FirstClass" University Project (CPU2018GF05) of China Pharmaceutical University, and the Fundamental Research Funds (2632018ZD08) for the Central Universities. We thank all technicians of Pharmaceutical Animal Experimental Center in China Pharmaceutical University for their kind help with in vivo experiments.

\section{Disclosure}

The authors report no conflicts of interest in this work.

\section{References}

1. Ganesh K, Wu C, O'Rourke K, et al. A rectal cancer organoid platform to study individual responses to chemoradiation. Nat Med. 2019;25(10):1607-1614. doi:10.1038/s41591-019-0584-2

2. O'Keefe S, Li J, Lahti L, et al. Fat, fibre and cancer risk in African Americans and rural Africans. Nat Commun. 2015;6(1):6342. doi: $10.1038 /$ ncomms 7342

3. Benson A, Venook A, Al-Hawary M, et al. NCCN guidelines insights: colon cancer, version 2.2018. J Natl Compr Canc Netw. 2018;16(4):359-369. doi:10.6004/jnccn.2018.0021

4. Lee S, Kim C, Sung S, et al. RIPL peptide-conjugated nanostructured lipid carriers for enhanced intracellular drug delivery to hepsin-expressing cancer cells. Int $J$ Nanomedicine. 2018;13:3263-3278. doi:10.2147/IJN.S166021

5. Demain A, Vaishnav P. Natural products for cancer chemotherapy. Microb Biotechnol. 2011;4(6):687-699. doi:10.1111/j.1751-7915.20 10.00221.x

6. Tan N, Zhou J. Plant cyclopeptides. Chem Rev. 2006;106(3):840-895. doi:10.1021/cr040699h

7. Morita H, Yamamiya T, Takeya K, et al. New antitumor bicyclic hexapeptides, RA-XI, -XII, -XIII and -XIV from Rubia cordifolia. Chem Pharm Bull. 1992;40(5):1352-1354. doi:10.1248/cpb.40.1352

8. Leung H, Zhao S, Yue G, et al. RA-XII inhibits tumour growth and metastasis in breast tumour-bearing mice via reducing cell adhesion and invasion and promoting matrix degradation. Sci Rep. 2015;5 (1):16985. doi:10.1038/srep16985

9. Fan J, Su J, Peng Y, et al. Rubiyunnanins C-H, cytotoxic cyclic hexapeptides from Rubia yunnanensis inhibiting nitric oxide production and NF- $\mathrm{kB}$ activation. Bioorg Med Chem. 2010;18(23): 8226-8234. doi:10.1016/j.bmc.2010.10.019

10. Song L, Wang Z, Wang Y, et al. Natural cyclopeptide RA-XII, a new autophagy inhibitor, suppresses protective autophagy for enhancing apoptosis through AMPK/mTOR/P70S6K pathways in HepG2 cells. Molecules. 2017;22(11):1934. doi:10.3390/molecules22111934

11. Wang J, Wang J, Li L, et al. RA-XII, a bicyclic hexapeptidic glucoside isolated from Rubia yunnanensis Diels, exerts antitumor activity by inhibiting protective autophagy and activating Akt-mTOR pathway in colorectal cancer cells. J Ethnopharmacol. 2021;266:113438. doi:10.1016/j.jep.2020.113438

12. Guo D, Wang Y, Wang J, et al. RA-XII suppresses the development and growth of liver cancer by inhibition of lipogenesis via SCAP-dependent SREBP suppression. Molecules. 2019;24(9):1829. doi:10.3390/molecules 24091829

13. Wang Y, Guo D, He J, et al. Inhibition of fatty acid synthesis arrests colorectal neoplasm growth and metastasis: anti-cancer therapeutical effects of natural cyclopeptide RA-XII. Biochem Biophys Res Commun. 2019;512(4):819-824. doi:10.1016/j.bbrc.2019.03.088

14. Li C, Yang X, An J, et al. A near-infrared light-controlled smart nanocarrier with reversible polypeptide-engineered valve for targeted fluorescence-photoacoustic bimodal imaging-guided chemo-photothermal therapy. Theranostics. 2019;9(25):7666-7679. doi: $10.7150 /$ thno. 37047

15. Yu J, Hu F, Zhu Q, et al. PD-L1 monoclonal antibody-decorated nanoliposomes loaded with paclitaxel and P-gp transport inhibitor for the synergistic chemotherapy against multidrug resistant gastric cancers. Nanoscale Res Lett. 2020;15(1):59. doi:10.1186/s11671019-3228-z

16. Poudel K, Banstola A, Gautam M, et al. Redox/photo dual-responsive, self-targeted, and photosensitizer-laden bismuth sulfide nanourchins for combination therapy in cancer. Nanoscale. 2021;13(2):1231-1247. doi:10.1039/D0NR07736D 
17. Poudel K, Banstola A, Gautam M, et al. Macrophage-membranecamouflaged disintegrable and excretable nanoconstruct for deep tumor penetration. ACS Appl Mater Interfaces. 2020;12 (51):56767-56781. doi:10.1021/acsami.0c17235

18. Zhang L, Li Y, Che W, et al. AIE multinuclear Ir(III) complexes for biocompatible organic nanoparticles with highly enhanced photodynamic performance. Adv Sci. 2019;6(5):1802050. doi:10.1002/advs.201802050

19. Miao W, Kim H, Gujrati V, et al. Photo-decomposable organic nanoparticles for combined tumor optical imaging and multiple phototherapies. Theranostics. 2016;6(13):2367-2379. doi:10.7150/thno.15829

20. Carter K, Luo D, Razi A, et al. Sphingomyelin liposomes containing porphyrin-phospholipid for irinotecan chemophototherapy. Theranostics. 2016;6(13):2329-2336. doi:10.7150/thno.15701

21. Kawauchi K, Sugimoto W, Yasui T, et al. An anionic phthalocyanine decreases NRAS expression by breaking down its RNA G-quadruplex. Nat Commun. 2018;9(1):2271. doi:10.1038/s41467-018-04771-y

22. Hu D, Chen L, Qu Y, et al. Oxygen-generating hybrid polymeric nanoparticles with encapsulated doxorubicin and chlorin e6 for trimodal imaging-guided combined chemo-photodynamic therapy. Theranostics. 2018;8(6):1558-1574. doi:10.7150/thno.22989

23. Yang $\mathrm{G}, \mathrm{Xu} \mathrm{L}$, Chao $\mathrm{Y}$, et al. Hollow $\mathrm{MnO}_{2}$ as a tumor-microenvironment-responsive biodegradable nano-platform for combination therapy favoring antitumor immune responses. Nat Commun. 2017;8(1):902. doi:10.1038/s41467-017-01050-0

24. Zhu Y, Jia H, Pan G, et al. Development of a light-controlled nanoplatform for direct nuclear delivery of molecular and nanoscale materials. $J \mathrm{Am}$ Chem Soc. 2018;140(11):4062-4070. doi:10.1021/jacs.7b13672

25. Pan L, Liu J, Shi J. Cancer cell nucleus-targeting nanocomposites for advanced tumor therapeutics. Chem Soc Rev. 2018;47(18): 6930-6946. doi:10.1039/C8CS00081F

26. Zielonka J, Joseph J, Sikora A, et al. Mitochondria-targeted triphenylphosphonium-based compounds: syntheses, mechanisms of action, and therapeutic and diagnostic applications. Chem Rev. 2017;117(15):10043-10120. doi:10.1021/acs.chemrev.7b00042

27. Martinon F. Targeting endoplasmic reticulum signaling pathways in cancer. Acta Oncol. 2012;51(7):822-830. doi:10.3109/0284186X.20 12.689113

28. Jia H, Jiang Y, Zhu Y, et al. Plasma membrane activatable polymeric nanotheranostics with self-enhanced light-triggered photosensitizer cellular influx for photodynamic cancer therapy. J Control Release. 2017;255:231-241. doi:10.1016/j.jconrel.2017.04.030
29. Jia H, Zhu Y, Xu K, et al. Plasma membrane-anchorable photosensitizing nanomicelles for lipid raft-responsive and light-controllable intracellular drug delivery. J Control Release. 2018;286:103-113. doi:10.1016/j.jconrel.2018.07.027

30. Lin F, Bao Y, Wu F. Improving the phototherapeutic efficiencies of molecular and nanoscale materials by targeting mitochondria. Molecules. 2018;23(11):3016. doi:10.3390/molecules23113016

31. Mahalingam S, Ordaz J, Low P. Targeting of a photosensitizer to the mitochondrion enhances the potency of photodynamic therapy. ACS Omega. 2018;3(6):6066-6074. doi:10.1021/acsomega.8b00692

32. Zhang D, Wen L, Huang R, et al. Mitochondrial specific photodynamic therapy by rare-earth nanoparticles mediated near-infrared graphene quantum dots. Biomaterials. 2018;153:14-26. doi:10. 1016/j.biomaterials.2017.10.034

33. Naor D, Sionov R, Ish-Shalom D. CD44: structure, function, and association with the malignant process. Adv Cancer Res. 1997;71:241-319.

34. Lee J, Termsarasab U, Park J, et al. Dual CD44 and folate receptor-targeted nanoparticles for cancer diagnosis and anticancer drug delivery. J Control Release. 2016;236:38-46. doi:10.1016/j. jconrel.2016.06.021

35. Wei X, Senanayake T, Warren G, et al. Hyaluronic acid-based nanogel-drug conjugates with enhanced anticancer activity designed for the targeting of CD44-positive and drug-resistant tumors. Bioconjug Chem. 2013;24(4):658-668. doi:10.1021/bc300632w

36. Poudel K, Banstola A, Tran T, et al. Hyaluronic acid wreathed, trio-stimuli receptive and on-demand triggerable nanoconstruct for anchored combinatorial cancer therapy. Carbohydr Polym. 2020;249:116815. doi:10.1016/j.carbpol.2020.116815

37. Noh I, Lee D, Kim H, et al. Enhanced photodynamic cancer treatment by mitochondria-targeting and brominated near-infrared fluorophores. Adv Sci. 2017;5:1700481. doi:10.1002/advs.201700481

38. Zhang Q, Deng C, Fu Y, et al. Repeated administration of hyaluronic acid coated liposomes with improved pharmacokinetics and reduced immune response. Mol Pharm. 2016;13(6):1800-1808. doi:10.1021/ acs.molpharmaceut.5b00952

39. Li S, Liu R, Jiang $X$, et al. Near-infrared light-triggered sulfur dioxide gas therapy of cancer. ACS Nano. 2019;13(2):2103-2113. doi: $10.1021 /$ acsnano.8b08700
International Journal of Nanomedicine

\section{Publish your work in this journal}

The International Journal of Nanomedicine is an international, peerreviewed journal focusing on the application of nanotechnology in diagnostics, therapeutics, and drug delivery systems throughout the biomedical field. This journal is indexed on PubMed Central, MedLine, CAS, SciSearch ${ }^{\mathbb{R}}$, Current Contents ${ }^{\mathbb{R}} /$ Clinical Medicine, $^{2}$

\section{Dovepress}

Journal Citation Reports/Science Edition, EMBase, Scopus and the Elsevier Bibliographic databases. The manuscript management system is completely online and includes a very quick and fair peer-review system, which is all easy to use. Visit http://www.dovepress.com/ testimonials.php to read real quotes from published authors. 